\section{Estudo de Sobrevivência de uma \\ Coorte de Pessoas de 60 Anose \\ Mais no Município de Botucatu \\ (SP) - Brasil}

\section{Survival Study of a Sixty Year-Old and Older Cohort in Botucatu (SP) - Brazil}

Tania Ruiz

Departamento de Saúde Pública

Faculdade de Medicina de Botucatu

Universidade Estadual Paulista - UNESP

Distrito de Rubião Júnior, s/n

18618-970 - Rubião Júnior - Botucatu - SP

truiz@fmb.unesp.br

\section{Liciana Vaz de Arruda Silveira Chalita}

Departamento de Estatística

Instituto de Biociências

Campus de Botucatu - UNESP

\section{Marilisa Berti de Azevedo Barros}

Departamento de Saúde Coletiva

Faculdade de Medicina

Universidade Estadual de Campinas - UNICAMP

\section{Resumo}

O aumento proporcional do número de idosos na população tem motivado estudos no sentido de melhorar a qualidade de vida desta faixa etária através de políticas sociais e, entre elas, o planejamento em saúde. Com o objetivo de conhecer riscos de mortalidade para a população de sessenta anos e mais, um estudo de sobrevida foi realizado rastreando, no ano de 1992, os idosos participantes de um inquérito de morbidade referida realizado na cidade de Botucatu em $1983 / 84$. Foram localizados $89,6 \%$ destes idosos. Curvas de sobrevivência foram calculadas com o método de Kaplan-Meier e a análise de riscos, utilizando-se a Regressão Múltipla de Cox ajustando-se o modelo agregando as variáveis por blocos. Para o sexo masculino foram encontradas associadas, independentemente, ao aumento da mortalidade as seguintes categorias de variáveis: idade de 70 anos e mais: Hazard Ratio $(\mathrm{HR})=2,4(1,6-3,7)$; salário menor que um salário mínimo: $\mathrm{HR}=2,2(1,3-3,8)$; ter "outras rendas": HR=2,2 (1,3-3,9); ser o chefe da família ou seu cônjuge: $\mathrm{HR}=2,3(1,2-2,4)$; referência de doenças do aparelho circulatório: HR=1,6 $(1,1-2,4)$; referência de diabetes mellitus: $H R=3,0(1,3-7,0)$. Para o sexo feminino, foram encontradas associadas a idade de 70 anos e mais: $\mathrm{HR}=4,6(3,0-7,1)$; referência de diabetes mellitus: $\mathrm{HR}=3,0(1,7-$ $5,3)$ e ter "outras rendas": HR=2,0 $(1,1-4,0)$.

Palavras-chave: Sobrevivência. Idoso. Mortalidade. 


\section{Introdução}

In order to determine mortality predictors among individuals aged 60 years and over, a survival study was conducted in 1992 by tracking a population of elders who participated in a general self-rated morbidity survey in 1983/84, in Botucatu, SP - Brazil. KaplanMeier estimates and the Cox proportional hazards method, available in SPSS and SAS statistical packages (v. 6.12), were used to analyze survival. The following mortality predictors were found for males $(\mathrm{p}<0.05)$ : being 70 years or older: Hazard Ratio $(\mathrm{HR})=2.4(1.6-3.7)$; earning one or less than one minimum wage: $\mathrm{HR}=2.2(1.3-3.8)$; having other income: $\mathrm{HR}=2.2$ (1.3-3.9); being the head of the household: HR=2.3 (1.2-2.4); self-rated morbidity due to circulatory diseases: HR=1.6 (1.1 - 2.4) and diabetes mellitus: HR=3.0 (1.3 - 7.0). Mortality predictors for females were the following: being 70 years or older: HR (4.6 (3.0 - 7.1); self-rated morbidity due to diabetes mellitus: $\mathrm{HR}=3.0$ (1.7-5.3); and having other income: $\mathrm{HR}=2.0(1.1-4.0)$.

Keywords: Survival. Aged. Mortality.
O aumento na proporção de idosos na população tem sido um dos fatos demográficos mais relevantes das últimas décadas, o que implica em novas demandas para as políticas sociais e de saúde. Os problemas de saúde que afetam os idosos são em geral de natureza crônica, e o desenvolvimento de ações efetivas de controle e prevenção para enfrentá-los constitui um desafio para os sistemas de atenção à saúde e uma prioridade no planejamento social. Estudos apontam que é possível melhorar a qualidade de vida dos idosos e evitar ou postergar as mortes que os atingem com a identificação de fatores de risco de mortes precoces e a atuação sobre eles com medidas de promoção e prevenção ${ }^{1,2}$. Idosos pertencentes a grupos de risco, identificados pela presença de fatores, deveriam merecer atenção especial dos programas sociais e de saúde. O conhecimento dos fatores associados à menor sobrevida pode orientar amplos programas educativos para a difusão de condições e estilos de vida mais saudáveis. Têm sido descritos na literatura como relacionados ao risco da mortalidade de idosos: fatores demográficos ${ }^{3}$, socioeconômicos ${ }^{4,5}$, de suporte social ${ }^{6}$, antecedentes de morbidade ${ }^{1,2,7}$, estilo de vida e hábitos ${ }^{8}$, entre outros.

O presente estudo objetivou descrever a sobrevivência segundo fatores de risco, de pessoas com sessenta anos ou mais, residentes em Botucatu, cidade de porte médio do interior do Estado de São Paulo. São poucos os estudos brasileiros de sobrevivência de idosos já publicados e avalia-se que o efeito de situações de risco sobre esta sobrevivência dependeria das características da cidade

\section{Material e Métodos}

Uma coorte de 640 pessoas com 60 anos ou mais, que fizeram parte da amostra de um inquérito de base populacional realizado em 1983 e 1984, foi rastreada em 1992 para verificar o status vital. A cidade contava na época do inquérito com 14.496 domicílios e 64.476 habitantes, dos quais $5.853(9,09 \%)$ 
tinham 60 anos ou mais. A amostra, estratificada e realizada em múltiplos estágios, incluiu 1.769 domicílios e 7.075 indivíduos, dos quais 640 eram pessoas com sessenta anos ou mais. No procedimento amostral, os setores censitários do município foram classificados em 5 estratos socioeconômicos, segundo características das moradias e de saneamento básico. A amostragem proporcional e aleatória garantiu que o número de domicílios dos estratos se mantivessem na amostra na mesma proporção do universo. Foram sorteados os estratos, e posteriormente houve seleção aleatória dos domicílios $^{9}$.

Em 1992, o rastreamento dos idosos incluiu: visita ao endereço que constava no inquérito de 83/84, busca de informação na vizinhança, uso de listas telefônicas, listas de grupos de idosos, prontuários dos hospitais e do Centro de Saúde Escola, relações de aposentados da Previdência e checagem de todas as declarações de óbitos ocorridas no município de Botucatu de 1984 a 1992.

$\mathrm{O}$ rastreamento possibilitou encontrar 574 dos 640 idosos estudados no inquérito, representando $89,6 \%$ da amostra inicial. Foram perdidos 66 casos, para os quais não se localizou uma declaração de óbito nem o possível paradeiro.

O inquérito de 1983/84 levantou informações sobre um conjunto de variáveis demográficas, socioeconômicas e de morbidade. As seguintes variáveis do inquérito foram redigitadas e analisadas neste estudo, pois poderiam constituir fatores de risco para a mortalidade dos idosos. Elas estão aqui arroladas, sendo que a categoria linha de base para a análise da sobrevida é a primeira a ser referida.

Variáveis demográficas e socioeconômicas: idade (60 a 69 anos; 70 anos ou mais), cor (branco; não branco), estar no mercado de trabalho (sim; não), salário (até um salário mínimo; um ou mais e menos de três salários mínimos; três ou mais salários mínimos), renda per capita (um ou mais salários mínimos; de 1/2 a um salário mínimo; menos que $1 / 2$ salário mínimo), outras rendas (possui; não possui), renda familiar (cinco ou mais salários mínimos; de três a cinco salários mínimos; até três salários mínimos) aposentado (sim; não), freqüentou escola (sim; não), tem automóvel (sim; não), tem telefone (sim; não), procedência (Botucatu; outro município, urbana de outro município, rural ???), tempo de residência no município (30 anos ou mais; de 15 a 29 anos; menos que 15 anos), condição de ocupação do domicílio (própria e alugada, outras formas).

- Variáveis relacionadas à família: casado (sim, não), chefe da família (o próprio ou o cônjuge, outros parentes), número de gerações na casa (uma, duas, três ou mais), número de pessoas na casa (uma ou duas, três ou quatro, cinco ou mais);

- Variáveis relacionadas à morbidade referida: doenças do aparelho circulatório, do aparelho respiratório, doenças hipertensivas, diabetes, neoplasias, doenças osteomusculares, outras doenças crônicas e presença de hipertensos na família (não, sim).

A análise dos dados incluiu, inicialmente, a descrição da coorte segundo o sexo. A seguir foi realizada a análise de sobrevivência, separadamente para cada sexo, utilizando-se o estimador produto-limite de Kaplan Méier ${ }^{10}$ no cálculo das curvas de sobrevivência, e o teste log-rank na comparação das mesmas. A análise conjunta dos fatores de risco para o óbito foi feita utilizando-se o modelo de riscos proporcionais de $\operatorname{Cox}^{11}$. Para este modelo manteve-se a categorização das variáveis da forma utilizada para o Kaplan-Meier, inclusive a idade.

Antes de incluir cada variável no modelo de Regressão de Cox foi testada a sua proporcionalidade através da análise do resíduo, utilizando-se o pacote estatístico SAS (v. 6.12) ${ }^{12}$. Para efetuar o ajuste dos modelos, a proporcionalidade foi verificada observando-se o comportamento das curvas de sobrevivência. As variáveis para as quais se verificou a não proporcionalidade dos riscos foram descartadas na confecção do modelo final multivariado. $\mathrm{O}$ estimador de Kaplan-Meier e o log-rang teste foram calculados para todas as variáveis e apresenta- 
dos os resultados para as variáveis encontradas como significantes.

Para cada modelo, a seleção das variáveis foi efetuada de acordo com o procedimento descrito em Collet ${ }^{13}$, partindo-se das variáveis com riscos proporcionais e diferença significativa entre as curvas de sobrevivência $(p<0,05)$ e considerando-se, em seguida, as variáveis com riscos proporcionais e diferença não significativa entre as curvas de sobrevivência. A variável idade foi incluída em todos os modelos para que o seu efeito não confundisse a análise dos efeitos das demais variáveis. Foram também testadas as interações entre as variáveis. As variáveis contínuas foram utilizadas já categorizadas por não se haver encontrado diferenças significantes nos resultados para a confecção do modelo de Cox.

Ajustou-se um modelo para cada bloco de variáveis e, a partir das variáveis selecionadas nesses três modelos, ajustou-se o modelo final, no qual foram incluídas, nesta ordem, as variáveis socioeconômicas, aquelas relacionadas à família e as de morbidade.

A análise foi feita separadamente para cada sexo, pois os segmentos feminino e masculino da população apresentam comportamentos bastante distintos com relação à sobrevida.

Os programas estatísticos utilizados para

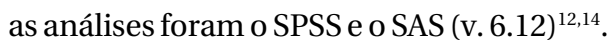

\section{Resultados}

Dosidosos de 1983/84 rastreados em 1992, $374(65,1 \%)$ estavam vivos e 200 haviam falecido. Dos 374 sobreviventes, 165 eram do sexo masculino e 209 do feminino; e dos falecidos, 101 eram do sexo masculino e 99 do sexo feminino (Tabela 1). Estes dados indicam que a incidência acumulada de óbitos desta coorte durante o período de 9 anos foi de $38 \%$ entre os homens e de $32,1 \%$ entre as mulheres.

Mais da metade dos idosos $(61,6 \%)$ estava na faixa de 60 a 69 anos, com um perfil demográfico que pode ser descrito como de um idoso branco $(89,2 \%)$, os homens casados $(84,6 \%)$ e as mulheres não $(56,8 \%)$, a metade deles morando no município há mais de 15 anos. A maioria (70,9\%) vivia com uma ou duas gerações na casa, sendo o idoso o chefe da família ou seu cônjuge (86,7\%). Viviam de aposentadorias ou pensões e tinham ainda baixa escolaridade e baixa renda (Tabela 1).

Em relação à saúde, 69,7 \% dos idosos referiram ter alguma doença crônica (com mais de um ano de duração); 36,9\% referiram ter alguma doença do aparelho circulatório, 23,7\% referiram ser hipertensos e 6,6\% referiram ter diabetes mellitus (Tabela 2).

Na análise de sobrevivência, as variáveis que apresentaram riscos proporcionais e diferenças estatisticamente significativas $(\mathrm{p}<0,05)$ no sexo masculino foram: idade, estar ou não no mercado de trabalho, estar ou não aposentado, chefia da família ou cônjuge, salário, "outras rendas", propriedade de automóveis e referência de doença do aparelho circulatório, diabetes mellitus e hipertensão (Tabelas 3 e 4). As variáveis que apresentaram riscos proporcionais e diferenças não significativas $(p>0,05)$ entre as curvas do sobrevivência foram: referência de hipertensão na família, de doença do aparelho respiratório e de doença do aparelho osteomuscular (Tabela 3).

Para o sexo feminino, as variáveis que apresentaram riscos proporcionais e diferença significativa entre as curvas de sobrevivência foram: idade, estado conjugal, chefia da família ou cônjuge e referência de diabetes mellitus (Tabela 4). As variáveis que apresentaram riscos proporcionais e diferença não significativa entre as curvas do sobrevivência foram: "outras rendas" e referência de doenças crônicas (Tabela 5).

$\mathrm{Na}$ análise múltipla dos fatores de risco (Tabelas 5 e 6), as categorias das variáveis que se mantiveram associadas à maior mortalidade das pessoas do sexo masculino foram: idade de 70 anos ou mais: Hazard Ratio $(H R)=2,4(1,6$ - 3,7), não ser o chefe da família: HR =2,3 $(1,2-4,4)$, não ter "outras rendas": HR =2,2 (1,3 - 3,9), salário menor que um salário mínimo: $\mathrm{HR}=2,2(1,3-3,8)$, haver referido diabete mellitus: $\mathrm{HR}=3,0(1,3-7,0)$ e haver referido doença do aparelho circulatório: HR =1,6 (1,1 - 2,4) (Tabela 5). 
Tabela 1 - Características sócio demográficas da coorte de idosos de 60 anos e mais, rastreada em 1992, segundo os dados de 1983/84, Botucatu, SP.

Table 1 - Demographic description of a cohort of elders aged sixty years and over, by sex. Botucatu (SP). 1983/84, 1992.

\begin{tabular}{|c|c|c|c|c|c|c|}
\hline \multirow[t]{2}{*}{ Variáveis } & \multicolumn{2}{|c|}{ Homens } & \multicolumn{2}{|c|}{ Mulheres } & \multicolumn{2}{|c|}{ Total } \\
\hline & No & (\%) & No & $(\%)$ & № & $(\%)$ \\
\hline Vivos ao final do estudo & 165 & $(62,0)$ & 209 & $(67,9)$ & 374 & $(65,2)$ \\
\hline \multicolumn{7}{|l|}{ Cor } \\
\hline brancos & 239 & $(89,8)$ & 273 & $(88,6)$ & 512 & $(89,2)$ \\
\hline não brancos & 27 & $(10,2)$ & 35 & $(11,4)$ & 62 & $(10,8)$ \\
\hline \multicolumn{7}{|l|}{ Estado conjugal } \\
\hline casados & 225 & $(84,6)$ & 133 & $(43,2)$ & 358 & $(62,4)$ \\
\hline não casados & 41 & $(15,4)$ & 175 & $(56,8)$ & 216 & $(37,6)$ \\
\hline \multicolumn{7}{|l|}{ Tempo residindo no município } \\
\hline até quinze anos & 52 & $(19,5)$ & 57 & $(19,5)$ & 109 & $(19,0)$ \\
\hline igual a quinze anos e mais & 214 & $(80,4)$ & 251 & $(81,5)$ & 465 & $(81,0)$ \\
\hline \multicolumn{7}{|l|}{ Aposentadoria } \\
\hline "não aposentados" & 74 & 27,8 & 198 & 64,3 & 272 & 47,4 \\
\hline "aposentados" & 191 & 71,8 & 110 & 35,7 & 301 & 52,5 \\
\hline sem informação & 1 & 0,4 & - - & - - & 1 & 0,01 \\
\hline \multicolumn{7}{|l|}{ Número de gerações na casa } \\
\hline só uma geração & 91 & 34,2 & 110 & 35,7 & 201 & 35,0 \\
\hline duas gerações & 103 & 38,7 & 103 & 33,4 & 206 & 35,9 \\
\hline três ou mais & 72 & 27,1 & 95 & 30,8 & 167 & 29,1 \\
\hline \multicolumn{7}{|l|}{ Chefia da família ou cônjuge } \\
\hline $\operatorname{sim}$ & 249 & 93,6 & 249 & 80,8 & 498 & 86,7 \\
\hline não & 17 & 6,4 & 59 & 19,2 & 76 & 13,3 \\
\hline \multicolumn{7}{|l|}{ Escolaridade } \\
\hline curso primário ou mais & 107 & $(40,2)$ & 80 & $(26,0)$ & 187 & $(32,6)$ \\
\hline primário incompleto e alfabetizados & 120 & $(45,1)$ & 112 & $(36,4)$ & 232 & $(40,1)$ \\
\hline analfabetos & 39 & $(14,7)$ & 116 & $(37,6)$ & 155 & $(27,0)$ \\
\hline \multicolumn{7}{|l|}{ Renda per capita (em salário mínimo) } \\
\hline igual a um SM ou mais & 135 & $(50,8)$ & 139 & $(45,1)$ & 274 & $(47,7)$ \\
\hline igual a $1 / 2$ e menos de um SM & 80 & $(30,1)$ & 107 & $(34,7)$ & 187 & $(32,6)$ \\
\hline até $1 / 2$ SM & 49 & $(18,4)$ & 57 & $(18,5)$ & 106 & $(18,5)$ \\
\hline sem informação & 2 & $(0,8)$ & 5 & $(1,6)$ & 7 & $(0,01)$ \\
\hline \multicolumn{7}{|l|}{ "Outras rendas" } \\
\hline possuíam outras rendas & 70 & $(26,3)$ & 42 & $(13,6)$ & 109 & $(18,9)$ \\
\hline não possuíam outras rendas & 186 & $(69,9)$ & 266 & $(86,4)$ & 451 & $(78,6)$ \\
\hline sem informação & 10 & $(3,7)$ & 4 & $(1,3)$ & 14 & $(2,4)$ \\
\hline
\end{tabular}

Tabela 2 - Morbidade referida (dados de 1983/84) em coorte de idosos de 60 anos e mais, rastreada em 1992, segundo o sexo. Botucatu (SP).

Table 2 - Blood pressure and self-reported morbidity of a cohort of elders (sixty years and over), by sex. Botucatu (SP). 1983/84.

\begin{tabular}{|c|c|c|c|}
\hline Variáveis & $\begin{array}{l}\text { Homens } \\
\mathrm{n}(\%)\end{array}$ & $\begin{array}{l}\text { Mulheres } \\
\mathrm{n}(\%)\end{array}$ & $\begin{array}{l}\text { Total } \\
\text { n (\%) }\end{array}$ \\
\hline \multicolumn{4}{|c|}{ Referência de doença cardiovascular } \\
\hline $\operatorname{sim}$ & $75(28,2)$ & $137(44,5)$ & $212(36,9)$ \\
\hline não & $191(71,8)$ & $171(55,5)$ & $362(63,1)$ \\
\hline \multicolumn{4}{|c|}{ Referência de hipertensão } \\
\hline $\operatorname{sim}$ & $43(16,2)$ & $93(30,2)$ & $136(23,7)$ \\
\hline não & $223(83,8)$ & $215(69,8)$ & $438(76,3)$ \\
\hline \multicolumn{4}{|c|}{ Referência de doença crônica } \\
\hline $\operatorname{sim}$ & $164(61,7)$ & $236(76,6)$ & $400(69,7)$ \\
\hline não & $102(38,3)$ & $72(23,4)$ & $174(30,3)$ \\
\hline \multicolumn{4}{|c|}{ Referência de diabetes mellitus } \\
\hline $\operatorname{sim}$ & $11(4,1)$ & $27(8,8)$ & $38(6,6)$ \\
\hline não & $255(95,9)$ & $281(91,2)$ & $536(93,4)$ \\
\hline
\end{tabular}


Tabela 3 - Tamanho da amostra (n), número de mortes (d) e resultado do teste log-rank para comparação de curvas de sobrevivência à mortalidade geral, estimadas pelo Método de Kaplan-Meier, para variáveis sociais e de morbidade que se mostraram proporcionais. Coorte de idosos de 60 anos e mais, do sexo masculino. 1983-1992. Botucatu (SP).

Table 3 -Sample ( $n$ ), deaths (d), and log-rank test for comparing survival curves with the general mortality estimated by the Kaplan-Meier Method, for proportional social and morbidity variables. Cohort of males, 60 years old and over. 1983-1992. Botucatu(SP).

\begin{tabular}{|c|c|c|c|}
\hline Variável & $\mathrm{n}$ & $d$ & valor de $p$ \\
\hline \multicolumn{4}{|l|}{ Idade } \\
\hline 60 a 69 anos & 176 & 46 & $<0,01$ \\
\hline 70 anos e mais & 90 & 55 & \\
\hline \multicolumn{4}{|l|}{ Salário } \\
\hline três ou mais salários mínimos & 87 & 23 & $<0,01$ \\
\hline um ou mais e menos de três salários mínimos & 103 & 39 & \\
\hline até um salário mínimo & 76 & 39 & \\
\hline \multicolumn{4}{|l|}{ Propriedade de automóvel } \\
\hline sim & 101 & 27 & $<0,01$ \\
\hline não & 165 & 74 & \\
\hline \multicolumn{4}{|l|}{ Outras rendas } \\
\hline $\operatorname{sim}$ & 70 & 15 & $<0,01$ \\
\hline não & 186 & 85 & \\
\hline \multicolumn{4}{|l|}{ Aposentado } \\
\hline $\operatorname{sim}$ & 74 & 17 & $<0,01$ \\
\hline não & 191 & 84 & \\
\hline \multicolumn{4}{|l|}{ Chefia da família } \\
\hline $\operatorname{sim}$ & 249 & 88 & $<0,01$ \\
\hline não & 17 & 13 & \\
\hline \multicolumn{4}{|l|}{ Estar no mercado de trabalho } \\
\hline $\operatorname{sim}$ & 64 & 17 & 0,02 \\
\hline não & 202 & 84 & \\
\hline
\end{tabular}

Para a população feminina, as categorias das variáveis que se mantiveram associadas à maior mortalidade foram: idade de $70 \mathrm{ou}$ mais: HR: 4,6 (3,0 - 7,1), não ter "outras rendas": HR =2,0 $(1,1-4,0)$ e referência de diabete mellitus: $\mathrm{HR}=2,0(1,1-4,0)$ (Tabela 6). Para as mulheres, nenhuma variável relacionada com a família se mostrou significativa quando "ajustada" para o efeito das demais.

\section{Discussão e Conclusão}

Os resultados deste estudo apontam que o nível sócio econômico (NSE) discrimina riscos diferenciados de mortalidade na coorte de idosos. Pertencer a NSE inferior (identificado por baixo salário e/ou por não dispor de outras rendas) significou um aumento de cerca de 2 vezes no risco de mortalidade dos idosos de ambos os sexos. Estes achados são coerentes com dados da litera- tura ${ }^{15}$ que apontam que, embora as diferenças socioeconômicas se expressem com maior intensidade na mortalidade de crianças, adolescentes e adultos jovens, a desigualdade social persiste, ainda que em menor grau, na mortalidade de idosos ${ }^{4,16}$.

Observou-se também que os homens que viviam em famílias das quais já não eram os chefes apresentaram risco de mortalidade igual a 2,3 vezes o risco daqueles que eram os chefes, independente da idade, do NSE e da morbidade referida. Continuar assumindo o papel de chefe da família pode representar situação que mantém a auto-estima e a satisfação pessoal. Embora tenha sido ajustado para as variáveis de nível econômico e para morbidade referida, é também necessário ponderar que inúmeras situações podem provocar a perda da chefia da família, tais como impossibilidade financeira, problemas e deficiências de saúde próprios ou de familiar e mesmo perda de familiar, entre 
Tabela 4 - Tamanho da amostra (n), número de mortes (d) e resultado do teste log-rank para comparação de curvas de sobrevivência à mortalidade geral, estimadas pelo Método de Kaplan-Meier, para variáveis que se mostraram proporcionais. Coorte de idosos de 60 anos e mais, do sexo feminino. 1983-1992. Botucatu (SP).

Table 4-Sample ( $n$ ), deaths (d), and log-rank test for comparing survival curves with the general mortality estimated by the Kaplan-Meier Method, for proportional variables. Cohort of females, 60 years old and over. 1983-1992. Botucatu(SP).

\begin{tabular}{|c|c|c|c|}
\hline Variável & $\mathrm{n}$ & $d$ & valor de $p$ \\
\hline \multicolumn{4}{|l|}{ Idade } \\
\hline 60 a 69 anos & 179 & 31 & $<0,0001$ \\
\hline 70 anos e mais & 129 & 68 & \\
\hline \multicolumn{4}{|l|}{ Èstar casada } \\
\hline $\operatorname{sim}$ & 133 & 34 & 0,023 \\
\hline não & 175 & 65 & \\
\hline \multicolumn{4}{|c|}{ Ser chefe da família ou sua cônjuge } \\
\hline $\operatorname{sim}$ & 249 & 73 & 0,012 \\
\hline não & 59 & 26 & \\
\hline \multicolumn{4}{|l|}{ Ter“Outras Rendas” } \\
\hline $\operatorname{sim}$ & 39 & 10 & 0,25 \\
\hline não & 266 & 89 & \\
\hline \multicolumn{4}{|c|}{ Referência de diabete mellitus } \\
\hline $\operatorname{sim}$ & 281 & 84 & 0,001 \\
\hline não & 27 & 15 & \\
\hline \multicolumn{4}{|c|}{ Referência de doenças crônicas } \\
\hline $\operatorname{sim}$ & 72 & 20 & 0,24 \\
\hline não & 236 & 79 & \\
\hline \multicolumn{4}{|c|}{ Referência de doença do aparelho circulatório } \\
\hline $\operatorname{sim}$ & 191 & 63 & $<0,01$ \\
\hline não & 75 & 38 & \\
\hline \multicolumn{4}{|c|}{ Referência de diabete mellitus } \\
\hline $\operatorname{sim}$ & 255 & 93 & $<0,01$ \\
\hline não & 11 & 8 & \\
\hline \multicolumn{4}{|c|}{ Referência de hipertensão } \\
\hline $\operatorname{sim}$ & 223 & 79 & 0,02 \\
\hline não & 43 & 22 & \\
\hline \multicolumn{4}{|c|}{ Referência de hipertensão na família } \\
\hline $\operatorname{sim}$ & 81 & 29 & 0,32 \\
\hline não & 61 & 27 & \\
\hline \multicolumn{4}{|c|}{ Referência de doença do aparelho respiratório } \\
\hline $\operatorname{sim}$ & 217 & 79 & 0,17 \\
\hline não & 49 & 22 & \\
\hline \multicolumn{4}{|c|}{ Referência de doença do aparelho osteomuscular } \\
\hline $\operatorname{sim}$ & 227 & 90 & 0,18 \\
\hline não & 39 & 11 & \\
\hline
\end{tabular}

outras. O fato de já não ter a chefia da família poderia desta forma não ser a causa do aumento do risco de óbito, mas uma conseqüência de outros determinantes desse aumento. Vale observar que, para as mulheres ser ou não cônjuge do chefe da família não implicou em mudanças no risco de mortalidade, indicando diferença de gênero no impacto da posição ocupada na família sobre a saúde dos idosos.

Este estudo revelou também que ter 70 anos ou mais significou uma probabilidade 
Tabela 5 - Resultados do ajuste do Modelo de Regressão de Cox aos dados de sobrevivência à mortalidade geral de uma coorte de idosos do sexo masculino com 60 anos ou mais. Botucatu, SP, 1983-1992.

Table 5 - Adjustment of Cox Regression for survival data to general mortality of a male cohort of individuals 60 years old and over. Botucatu, SP. 1983-1992.

\begin{tabular}{lc}
\hline Variável & $\begin{array}{c}\text { Razão dos Riscos } \\
\text { (intervalo de confiança - 95\%) }\end{array}$ \\
\hline Idade & 1,0 \\
$\quad 60$ a 69 anos & $2,4(1,6-3,7)$ \\
$\quad 70$ anos e mais & 1,0 \\
Salário & $1,3(0,7-2,1)$ \\
$\quad$ Igual ou mais de três salários mínimos & $2,2(1,3-3,8)$ \\
$\quad$ Igual a um até menos de três salários mínimos & \\
$\quad$ Até um salário mínimo & 1,0 \\
Ter “Outras Rendas” & $2,2(1,3-3,9)$ \\
$\quad$ Sim & 1,0 \\
$\quad$ Não & \\
Ser o chefe da família ou seu cônjuge & $2,3(1,2-4,4)$ \\
$\quad$ Sim & \\
$\quad$ Não & 1,0 \\
Referência de doenças do aparelho circulatório & $1,6(1,1-2,4)$ \\
$\quad$ Não & 1,0 \\
Sim & $3,0(1,3-7,0)$ \\
Referência de diabete mellitus & \\
$\quad$ Não &
\end{tabular}

Tabela 6 - Resultados do ajuste do Modelo de Regressão de Cox aos dados de sobrevivência à mortalidade geral de uma coorte de idosos do sexo feminino com 60 anos ou mais. Botucatu, SP, 1983-1992.

Table 6 - Adjustment of Cox Regression for survival data to general mortality of a female cohort of individuals 60 years old and over. Botucatu, SP, 1983-1992.

\begin{tabular}{lc}
\hline Variável & $\begin{array}{c}\text { Razão dos Riscos } \\
\text { (intervalo de confiança - 95\%) }\end{array}$ \\
\hline Idade & 1,0 \\
$\quad 60$ a 69 anos & $4,6(3,0-7,1)$ \\
70 anos e mais & 1,0 \\
Referência de diabetes mellitus & $3,0(1,7-5,3)$ \\
Não & 1,0 \\
Sim & \\
Ter “Outras Rendas” & $2,0(1,1-4,0)$ \\
Sim & \\
Não &
\end{tabular}

de morte para os homens, durante os 8 anos de seguimento, 2,4 vezes a daqueles com 60 a 69 anos. e 4,6 vezes [FALTA ALGO NESTA FRASE!!!] Nas mulheres, a razão dos riscos foi de 4,6. A diferença destas razões entre os sexos decorre da maior média de idade das mulheres deste segmento etário (70 anos e mais) em comparação com os homens. 
Em relação à morbidade, os idosos que referiram ter diabetes apresentaram um risco de óbito 3 vezes maior que o risco dos não diabéticos. Morgan et al. ${ }^{1}$ encontraram, em estudo de coorte, que a taxa de mortalidade de diabéticos era 4 vezes a dos não diabéticos. Verificaram que os homens diabéticos perdiam em média 7 anos de vida, e as mulheres diabéticas 7,5 anos.

Entre os homens, a presença de doença cardiovascular referida significou um aumento de $60 \%$ no risco de mortalidade, comparativamente com aqueles que não tinham referido estas doenças. Psaty et al. ${ }^{2}$ observaram aumento do risco de mortalidade total de cerca de $20 \%$ em idosos hipertensos, em comparação com normotensos.

Embora neste estudo a presença de hipertensão (medida no inquérito em subgrupo da amostra) não tenha se mostrado associada a aumento estatisticamente significativo da probabilidade de morte, a presença de doença do aparelho circulatório, que inclui a referência de hipertensão, mostrou esta associação.

É preciso enfatizar para a interpretação adequada dos achados que trata-se de morbidade referida e não de diagnósticos clínicos confirmados por critérios especificados.

Os resultados deste estudo apontam para a presença de fatores que atuam elevando $o$ risco da mortalidade em idosos. Os resultados revelam-se coerentes com os observa- dos na literatura e indicam que é possível reduzir a mortalidade de idosos com intervenções especiais voltadas a este segmento da população. Estudos apontam o sucesso na aplicação de intervenções em comunidades levando à redução dos fatores e a comportamentos de risco para a saúde ${ }^{17}$. Modificações na dieta com aumento do consumo de frutas e verduras têm levado, segundo estudos, à redução de cerca de $20 \%$ da taxa de mortalidade total ${ }^{18}$.

Este estudo aponta ainda que os idosos de nível sócio econômico mais baixo e que apresentam doenças crônicas merecem atenção e programas especiais, com cuidados que de fato sejam efetivos no controle das doenças crônicas e na promoção de hábitos saudáveis, de auto-estima e de bemestar psíquico. Estudos verificam que a depressão e sintomas associados representam fatores de risco para o aumento da mortalidade de idosos ${ }^{19}$.

As ações dos programas de saúde estaduais e nacionais do Sistema Único de Saúde voltadas para os pacientes diabéticos precisam ganhar efetividade para o conjunto dos pacientes mais idosos e assumir prioridade, especialmente em municípios com alta proporção de idosos, como é o caso de Botucatu e tantos outros municípios brasileiros. A evidência destes fatos nos alerta para a importância de perseguir metas de promoção da saúde dos idosos na programação de saúde local.

\section{Referências}

1. Morgan CL, Currie CJ, Peters JR. Relationship between diabetes and mortality: a population study using record linkage. Diabetes Care 2000; 23(8): 1103-7.

2. Psaty BM, Furberg CD, Kuller LH, Cushman M et al. Association between blood pressure level and the risk of myocardial infarction, stroke and total mortality: the cardiovascular health study. Arch Intern Med 2001; 161(9): 1183-92.

3. Spino, DV, Parra, EO, Kriehbiel, R. Mortality differences between elderly mexican americans and non-hispanic whites in San Antonio, Texas. J Am Geriatr Soc 1994; 42: 604-8.
4. Black D, Morris JN, Smith C, Townsend P et al. Inequalities in Health: The Black Report: The Health Divide. London: Penguin; 1988.

5. Martelin T. Mortality by indicators of socioeconomic status among the Finnish elderly. Soc Sci Med 1994; 38: 1257-78.

6. Steinbach U. Social networks, institutionalization, and mortality among elderly people in the United States. $J$ Gerontol 1992; 47: S183-90. 
7. Balkau B, Shipley M, Jarret RJ et al. High blood glucose concentration is a risk factor for mortality in middleaged nondiabetic men. Diabetes Care 1998; 21(3): 360-7.

8. Fried LP, Kronmal RA, Newman AB et al. Risk factors for 5-year mortality in older adults. The Cardiovascular Health Study. JAMA 1988; 279: 585-92.

9. Carandina L, Sanches O, Carvalheiro JR. Análise das condições de saúde e de vida da população urbana de Botucatu.(SP). I. Descrição do plano amostral e avaliação da amostra. Rev Saúde Pública 1986; 20: 465-74.

10. Kaplan EL, Meier P. Nonparametric estimation from incomplete observation. J Am Stat Assoc 1958; 53: 45781 .

11. Cox DR. Regression models and life tables (with discussion). J R Stat Soc B 1972; 43: 187-220.

12. SAS Institute. SAS Introdutory guide for personal computers, release 6.03 edition. USA. SAS Institute Inc., 1988.

13. Collet D. Modelling survival data in medical research. London: Champman \& Hall; 1994.

14. Norussis M J. Statistical Package for Social Science (SPSS) for Windows Advanced Statistics Release 5.0. Chicago: SPSS;1992.
15. Burke GL, Arnold AM, Bild DE, Cushman M et al. Factors associated with healthy aging: the cardiovascular health study. J Am Geriatr Soc 2001; 49(3): 254-62.

16. Lynch JW, Kaplan GA, Panmuk ER, Cohen RD et al. Income inequality and mortality in Metropolitan areas of the United States. Am J Public Health 1998; 88(7): 1074-80.

17. Scheuermann W, Razum O, Scheidt R, Wiesemann A et al. Effectiveness of a decentralized, community related approach to reduce cardiovascular disease risk factor levels in Germany. Eur Heart J 2000; 21(19): 1591-7.

18. Khaw KT, Bingham S, Welch A, Luben R et al. Relation between plasma ascorbic acid and mortality in men and women in EPIC-Norfolk prospective study: a prospective population study. European Prospective Investigation into Cancer and Nutrition. Lancet 2001; 357(9257): 657-63.

19. Pulska T, Pahkala K, Laippala P, Kivela SL. Depressive symptoms predicting six-year mortality in depressed elderly finns. Int J Geriatr Psychiatry 2000; 15(10): 940-6.

Recebido em: 26/09/2001

Versão final reapresentada em: 22/02/2003

Aprovação em: 05/05/2003 